\title{
Peritoneal sarcoidosis: an unusual presentation and a brief review of the literature
}

\author{
A. Nicolini ${ }^{1}$, M. Vita 2 , S. Lanata ${ }^{3}$
}

ABSTRACT: Peritoneal sarcoidosis: an unusual presentation and a brief review of the literature. A. Nicolini, M. Vita, S. Lanata.

Peritoneal involvement continues to be a rare manifestation of sarcoidosis: its involvement is not always isolated and sarcoid granulomas are also found elsewhere. Peritoneal diseases tend to have an increased incidence in women. Peritoneal involvement presents as ascites, as peritoneal thickening and multiple soft tissue nodules, and can often simulate peritoneal carcinomatosis. We describe a case of a man pre- senting abdominal pain, nausea, vomiting and a clinical picture of intestinal obstruction, with peritoneal sarcoidosis and abdominal findings suggesting peritoneal carcinomatosis. The diagnosis of sarcoidosis was confirmed by biopsy of the peritoneum during surgical laparotomy. Peritoneal involvement is a rare manifestation of sarcoidosis (less than $\mathbf{3 0}$ cases described in English medical literature) : to our knowledge this is the first reported case of the disease presenting with an acute abdominal obstruction treated with surgery. Monaldi Arch Chest Dis 2011; 75: 2, 132-134.

Keywords: Peritoneal sarcoidosis, Intestinal obstruction, Surgical laparotomy.

1 Division of Respiratory Diseases, Hospital of Sestri Levante (Genova),

2 Division of General Surgery, Hospital of Lavagna (Genova),

3 Histopathology Service, Hospital of Sestri Levante (Genova), Italy.

Correspondence: Dr. Nicolini Antonello, Via Novara 5, 16039 Sestri Levante, Italy; e-mail:antonello.nicolini@fastwebnet.it

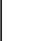

S

Sarcoidosis is a multisystemic idiopathic granulomatous disease: the lung is the most common organ involved by the disease, but any organ can be involved [1]. Serosal involvement is rarely reported, especially when it is the unique manifestation of the disease [2]. Only 36 cases were reported in the literature published in English until 2008 [3]. Peritoneal involvement can manifest as fluid accumulation within the peritoneal cavity (ascites) or soft tissue infiltration: Computed tomography (CT) is an excellent means of detecting and characterising the disease, which can frequently mimic malignancy [4]. Moreover CA 125 serum level may be elevated and this entity may be confused in several cases with ovarian carcinoma $[1,5]$. The presence of non-caseating granulomata suggests the diagnosis of sarcoidosis, but the absence of other causes of granulomatous disease, especially tuberculosis, must be documented [6]. Clinically there is abdominal pain, vomiting or more rarely hiccups and intestinal obstruction $[6,7]$ and sometimes a decrease in appetite and signs of ascites [8]; in three cases a rare occurrence of an association with pleural effusion has been described [9]. We shall describe the case of a man presenting with an adhesive small-bowel obstruction suggesting peritoneal neoplasm.
Case report
In January 2009 a 57 year old man, affected by chronic psychiatric illness, was taken to the Emergency Department presenting with complaints of left-sided abdominal pain, nausea and vomiting. In his past clinical history there was diabetes and diverticulosis of the colon. His abdomen was diffusely tender. X-ray film of the abdomen and water-soluble contrast transit time demonstrated adhesive small-bowel obstruction. An abdominal CT scan was performed and showed no ascites but peritoneal thickening and small size nodular enhancement (fig. 1). A CT scan of the thorax showed a solitary pulmonary nodule. Due to a persistent untreatable obstruction, a laparotomy was carried out. The abdominal picture found by surgeons was characterised by abdominal adhesions in addition to soft tissue nodules involving the peritoneum and described as being like a carcinomatosis. Laboratory findings were normal except C-reactive protein 1.00 (normal range 0-0.50) serum angiotensin-converting enzyme $67 \mathrm{U} / \mathrm{L}$ (normal range 18 - 55); CA 125 level was normal, as was blood cell count. The PPD tuberculin skin test was negative. Histopathologic findings consisted of non-caseating epithelioid cell granulomas, occurring individually or more rarely packed in an irregular nodular form. The granulomas were usu- 


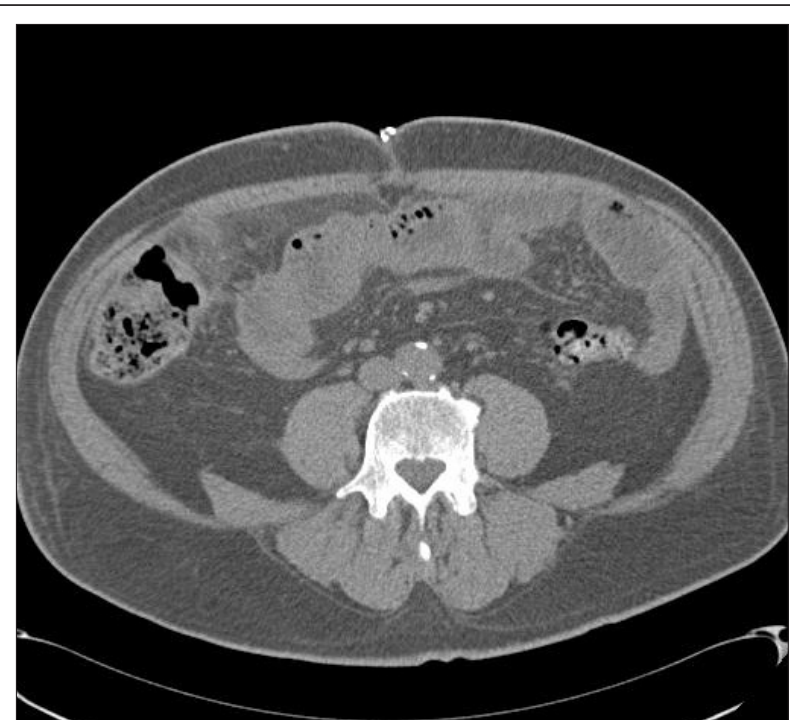

Fig. 1. - Abdominal CT scan: peritoneal thickening and nodular enhacement.

ally small and composed of multinucleated giant cells rarely containing cytoplasmatic inclusions (asteroid or Schaumann bodies), epithelioid histiocytes and few lymphocytes. Some of them were centrally occupied by nodular hyaline scar with or without fibrinoid necrosis (fig. 2). Special stains (Ziehl-Neelsen, Grocott and PAS) did not show the presence of an infectious agent. The granulomas appear both in the samples of the omentum and of the peritoneum. Pulmonary lung function tests were as follows: FVC $3.35 \mathrm{~L}(84.5 \%$ predicted) $\mathrm{FEV}_{1} 2.94 \mathrm{~L}$ (87.5\% predicted) $\mathrm{FEV}_{1} / \mathrm{FVC} 87.7$, DLCO $83.6 \%$. A needle lung biopsy was performed later and revealed the absence of neoplastic cells and a cytologic image was suggestive (but not conclusive) of sarcoidosis. The patient refused to undergo open lung biopsy or corticosteroid therapy. He did not attend the planned follow-up.

\section{Discussion}

Gastrointestinal involvement of sarcoidosis is rare (1\% of all cases) [10] and sarcoidosis of its serosal surface is reported even more rarely; presentation is between the second and fourth decade of life with an increased incidence in women [10]. Isolated peritoneal involvement appears in a small number of described cases as presented in a recent review of 36 cases [9]. The peritoneal cavity is a potential space enclosed by visceral and parietal peritoneum. This serosal membrane also forms the mesenteries, lines the omenta and partially or completely covers abdominal and pelvic viscera. The small-bowel mesentery, enclosed by folds of the peritoneum, extends diagonally from the duodenal junction in the left upper quadrant to the ileocecal valve in the right lower quadrant. This, in combination with a series of other mesenteries (trasverse mesocolon, sigmoid mesentery) and ligaments (coronary, gastrohepatic, hepatoduodenal, falciform, gastrocolic, splenorenal and phrenicolic), formed by peritoneal reflections and attachments, leads to anatomic compartmentalisation of the peritoneal cavity [11]. Overall, computed tomography (CT) is an excellent means of identifying the normal anatomy of the peritoneum and detecting and characterising peritoneal diseases. Peritoneal diseases are most commonly identified on the CT as ascites and/ or soft tissue infiltration of the peritoneal ligaments and mesenteries. Although a variety of relatively common peritoneal processes can be observed, most notably peritoneal carcinomatosis to gastrointestinal or ovarian tumours uncommon non-neoplastic conditions can also involve the peritoneal and subperitoneal spaces [4]. This different group of non-neoplastic conditions includes systemic diseases such as eosinophilic gastroenteritis and amyloidosis, tumour-like conditions such as aggressive fibromatosis or inflammatory pseudotumors, and atypical infections such as tuberculosis, Whipple disease and actinomycosis $[4,11]$. Sarcoidosis can be categorised as both systemic and granulomatous disease involving on rare occasions the peritoneum [7]. Laparotomy or laparoscopy is always required to reveal the involvement of the disease in the visceral and parietal peritoneum, because of the small size of the nodules, which may escape detection on the CT scan $[8,9,12]$. Peritoneal sarcoidosis should be considered in the differential diagnosis with tuberculosis, fungal infections and carcinomatosis: in several cases tumour marker CA 125 level is high and can arose suspicion of ovarian malignancy [5]. Peritoneal sarcoidosis is a very rare condition and it can be misinterpreted as a metastatic disease or endometriosis:an elevated CA 125 has been reported in connection with sarcoidosis and should be considered in determining the diagnosis, above all in the presence of predominantly lymphocytic ascitic liquid. Moreover, the exclusion of peritoneal tuberculosis is essential, even in the context of a negative PPD, because the disease is non-specific and may coincide with immunosuppression [13]. Most cases of peritoneal sarcoidosis usually have a benign course, evolving spontaneously or with a short course of corticosteroid therapy [3], sometimes with the addition of methotrexate [14].

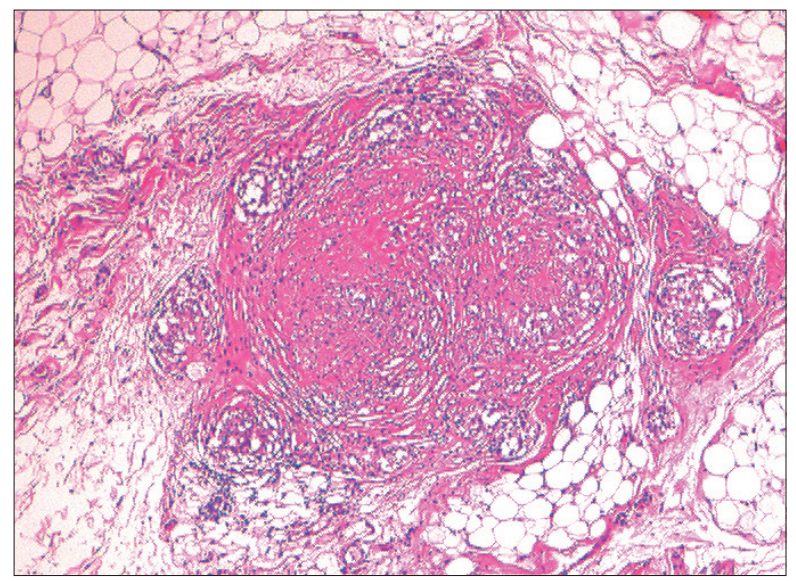

Fig. 2. - Histopathologic findings of peritoneum biopsy (hematoxilin and eosin $4 \mathrm{x}$ ). Histopathological pictures is characterized by non caseating epithelioid cells granulomas, that can occur singly or more rarely packed in an irregular nodular form. 
TNFo-blocking therapy has been proposed in the management of refractory sarcoidosis [14, 15] with discontinuous results. In short, we have described a rare case of isolated peritoneal sarcoidosis simulating peritoneal carcinomatosis and presenting with acute abdominal obstruction. When the isolated disease is present it has non- specific radiological imaging and only a biopsy may lead to a definitive diagnosis.

\section{References}

1. Judson MA Extrapulmonary sarcoidosis. Sem Respir Crit Care 2007; 28: 83-101.

2. Uthman IW, Bizri AN, Khalifeh. Peritoneal sarcoidosis. Sem Arthr Rheumat 2002; 31: 353.

3 Bourdillon L, Lanier-Gachon E, Stankovic K, Broussolle C, Seve P. Lofgren syndrome and peritoneal involvement by sarcoidosis. Chest 2007; 132: 310-312.

4. Pickhardt PJ, Bhalla S. Unusual non-neoplastic peritoneal and sub-peritoneal conditions: CT findings. $R a-$ diographics 2005: 25: 719-730.

5. Kalluri M, Judson MA. Sarcoidosis associated with an elevated serum CA 125 level: description of a case and review of the literature. Am J Med Sci 2007; 334: 441443.
6. Ngo' Y, Messing B, Marteau P, et al. Peritoneal sarcoidosis: an unrecognized cause of sclerosing peritonitis. Dig Dis Scien 1992; 37: 1776-1780.

7. Hackworth WA, Kimmelshue KN, Stravitz RT. Peritoneal sarcoidosis: a unique cause of ascites and intractable hiccups. Gastroent Hepatol 2009; 5: 859-861.

8. Otrock ZK, Oghlakian GO, Bizri AN. Peritoneal sarcoidosis. J Gastroenterol Hepatol 2005; 20: 1947.

9. Iyer S, Afshar K, Sharma OP. Peritoneal and pleural sarcoidosis: an unusual association: review and clinical report. Curr Opin Pulm Med 2008; 14: 481-487.

10. Costa F, Arrobas A. Sarcoidose extratoracica. Riv Port Pneumol 2008; 14: 127-140.

11. Lubner MG, Pickhardt PJ. Peritoneal sarcoidosis.the role of imaging in diagnosis. Gastroenterol Hepathol 2009; 5: 861-863.

12. Leleu O, Aron C, Lavallard B, Brunin G, Rohart P. Urgence chirurgicale relevant une sarcoidose.A propose d'un cas. Rev Pneumol Clin 1996; 52: 196-198.

13. Brown JV, Epstein HD, Chang M, Goldstein BH. Sarcoidosis presenting as an intraperitoneal mass. Case Rep Oncol 2010; 3: 9-13.

14. Dasilva V, Breuil V, Chevallier P, Euller-Ziegler L. Relapse of severe sarcoidosis with uncommon peritoneal location after TNF $\alpha$ blockade. Efficacy of rituximab: report of a single case. Joint Bone Spine 2010; 77: 82-83.

15. Doty JD, Mazur JE, Judson MA. Treatment of sarcoidosis with infiximab. Chest 2005; 127: 1064-1071.

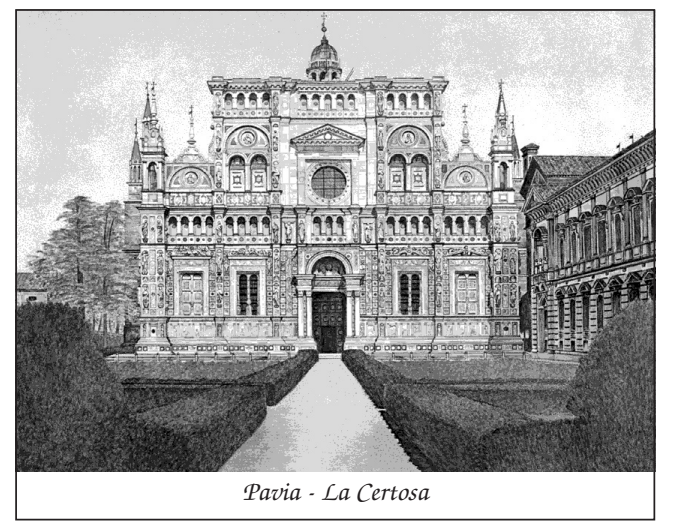

